\title{
Sympathetic Hyperactivity Influences Chemosensor FunCtion in Patients With END-Stage Renal Disease
}

\author{
C. Meyer ${ }^{1}$, P. Schueller ${ }^{1}$, J. Balzer ${ }^{1}$, T. Lauer ${ }^{1}$, R. Westenfeld ${ }^{1}$, P. Schauerte ${ }^{2}$, M. Hennersdorf ${ }^{3}$, \\ S. Steiner ${ }^{1}$, M. Kelm¹ ${ }^{1}$ T. Rassaf ${ }^{1}$ \\ ${ }^{1}$ Division of Cardiology, Pulmonology and Angiology, University of Duesseldorf, \\ ${ }^{2}$ Division of Cardiology and Pulmonology, RWTH Aachen University, \\ ${ }^{3}$ Medical Clinic I, SLK-Kliniken Heilbronn, Germany
}

\begin{abstract}
Background: Autonomic neuropathy is common in patients suffering from end-stage renal disease (ESRD). This may in part explain the high cardiovascular mortality in these patients. Chemosensory function is involved in autonomic cardiovascular control and is mechanistically linked to the sympathetic tone.

Objective: The aim of the present study was to assess whether sympathetic hyperactivity contributes to an altered chemosensory function in ESRD.

Material and methods: In a randomized, doublemasked, placebo controlled crossover design we studied the impact of chemosensory deactivation on heart rate, blood pressure and oxygen saturation in 10 ESRD patients and 10 age and gender matched controls. The difference in the R-R intervals divided by the difference in the oxygen pressures before and after deactivation of the chemoreceptors by 5-min inhalation of $7 \mathrm{~L}$ oxygen was calculated as the hyperoxic chemoreflex sensitivity (CHRS). Placebo consisted of breathing room air. Baseline sympathetic activity was characterized by plasma catecholamine levels and 24-h time-domain heart rate variability (HRV) parameters.

Results: Plasma norepinephrine levels were increased $(1.6 \pm 0.4$ vs. $5.8 \pm 0.6 ; \mathrm{P}<0.05)$ while the SDNN (standard deviation of all normal R-R intervals: 126.4 \pm 19 vs. $100.2 \pm 12 \mathrm{~ms}$ ), the RMSSD (square root of the mean of the squared differences between adjacent normal R-R intervals: $27.1 \pm 8$ vs. $15.7 \pm 2 \mathrm{~ms}$ ), and the 24 -h triangular index $(33.6 \pm 4$ vs. $25.7 \pm 3$; each $\mathrm{P}<0.05)$ were decreased in ESRD patients as compared to controls. CHRS was impaired in ESRD patients $(2.9 \pm 0.9 \mathrm{~ms} / \mathrm{mmHg}, \mathrm{P}<0.05)$ as compared to controls $(7.9 \pm 1.4 \mathrm{~ms} / \mathrm{mmHg})$. On multiple regression analysis 24 h-Triangular index, RMSSD, and plasma norepinephrine levels were independent predictors of an impaired hyperoxic CHRS.

Conclusion: Sympathetic hyperactivity influences chemosensory function in ESRD resulting in an impaired hyperoxic CHRS.
\end{abstract}

Key words: chemoreflexes, end-stage renal disease, nervous system, sympathetic

\section{INTRODUCTION}

Autonomic neuropathy is common in patients with end-stage renal disease (ESRD). This may in part explain the high cardiovascular mortality in these patients [1-4]. The underlying mechanisms of a sympathetic-parasympathetic imbalance in ESRD are still not fully understood. Previous reports demonstrated that sympathetic activation in ESRD involves firing of afferent renal nerve fibres, activation of the renin-angiotensin system, and decreased nitric oxide bioavailability [5]. A causal link between tonic chemoreflex activation and an increased efferent sympathetic activity to muscle circulation has recently been reported [6].

Deactivation of peripheral chemosensors by inhalation of pure oxygen, leads to sympathetic withdrawal and a subsequent decrease of heart rate $[6,7]$. Impairment of this desensitization, as a consequence of tonic chemoreflex activation, has been demonstrated to be a predictor of ventricular tachyarrhythmias. The hyperoxic cardiac chemoreflex sensitivity (CHRS) reflects the relative decrease of R-R intervals during administration of concentrated $\mathrm{O}_{2}$ and characterizes the deactivation of carotid and/or aortic chemoreceptors. The aim of the present study was to assess whether sympathetic hyperactivity influences chemosensory function in ESRD.

\section{Material AND Methods}

\section{STUdy Design}

The study was approved by a local Ethics Committee. In a randomized, double-masked, placebo controlled crossover design we studied the impact of chemosensory deactivation on heart rate, blood pressure, and oxygen saturation by 5 -min inhalation of $100 \%$ oxygen in 10 ESRD patients and 10 age and gender matched controls. We assessed whether the resting sympathetic tone, as measured by baseline plasma catecholamine levels and time-domain heart rate variability (HRV) parameters, contributes to chemosensory activation in ESRD resulting in an impaired hyperoxic CHRS. Placebo consisted of breathing room air. 


\section{PATIENTS}

Patients with ESRD (21 - 80 years) who had been on chronic hemodialysis for at least 6 months were investigated. Other cardiovascular risk factors and pre-existing cardiovascular disease did not preclude from participation in the study. Exclusion criteria were congestive heart failure with a cardiac ejection fraction of $<35 \%$, acute inflammation (C-reactive protein $>5$ $\mathrm{mg} / \mathrm{l}$ ), chronic pulmonary diseases, diabetes mellitus, neurocardiogenic syncope, sleep apnoea syndrome, hyperthyroidism, and heart rhythm other than sinus (Table 1).

Table 1. Baseline characteristics.

\begin{tabular}{lll}
\hline & $\begin{array}{l}\text { Controls } \\
(\mathrm{n}=10)\end{array}$ & $\begin{array}{l}\text { ESRD } \\
(\mathrm{n}=10)\end{array}$ \\
\hline Age, yr & $62 \pm 6$ & $65 \pm 4$ \\
Male sex, n & 6 & 6 \\
Hx hypertension, n & 7 & 7 \\
Hx dyslipidemia, n & 8 & 7 \\
Hx smoking, n & 6 & 5 \\
Hx CAD, n & 5 & 4 \\
Hx CVD, n & 4 & 1 \\
Hemoglobin, g/l & $132 \pm 7$ & $126 \pm 4$ \\
Medication, n (\%) & & \\
$\quad$ Alpha-blockers & 3 & 1 \\
Beta-blockers & 6 & 5 \\
ACE-inhibitors/ AT-II-antagonists & 6 & 6 \\
Calcium antagonists & 5 & 2 \\
Central sympatholytic agents & 0 & 2 \\
Isosorbide dinitrate & 3 & 1 \\
Diuretics & 7 & 5 \\
Statins & 7 & 5 \\
\hline
\end{tabular}

$\mathrm{Hx}$, indicates history of; CAD, coronary artery disease; CVD, cerebrovascular disease; Values are means \pm SE.

Measurements of Heart Rate, Blood Pressure, Standard Clinical BloOd Parameters, AND SYMPATHETIC ACTIVITY

Blood pressure was measured non-invasively with a sphygmomanometer cuff. Blood was drawn after a 30 min resting period through large-bore angiocatheters (18 gauge) to prevent artefact hemolysis. The first $3 \mathrm{ml}$ of blood was discarded, and the blood then slowly drawn into prechilled tubes. Standard clinical blood parameters including renal function were immediately analyzed in a central laboratory using standard techniques [8]. Plasma norepinephrine and epinephrine were quantified by high-performance liquid chromatography. The tubes were kept on ice until centrifugation at $4^{\circ} \mathrm{C}$. Plasma samples were stored at $-70^{\circ} \mathrm{C}$ until assayed. Heart rate was measured continuously by a 12-channel electrocardiogram (ECG). Additionally, the mean R-R interval was calculated from 10 consecutive R-R intervals. HRV time-domain measures were determined from an ambulatory 24-h ECG in the day without hemodialysis in order to avoid the influence of the hemodialysis session on HRV parameters. The following HRV parameters, which are considered to be standardized in the general population, were calculated for each patient to characterize cardiac sympathetic-parasympathetic control: (i) the 24-h SD of normal R-R intervals (SDNN, ms), as a global measure of HRV; (ii) The SD of the average normal R-R interval for all 5-min segments of a 24-h ECG recording (SDANN, ms); and (iii) the square root of the mean of the squared differences between adjacent normal R-R intervals (RMSSD, ms); (iv) The Triangular index, a time-domain geometric measure.

\section{Chemosensory Deactivation}

To characterize the effect of chemosensory deactivation on heart rate, blood pressure, and oxygen saturation, we determined the venous partial oxygen pressure $\left(\mathrm{pO}_{2}\right)$, before and after breathing oxygen and room air, respectively, via a nose mask. Baseline measurements were taken after patients were recumbent for a period of 10 min. Pure oxygen or room air was then administered via a nose mask for $5 \mathrm{~min}$. Following a 30 -min recovery, the identical protocol (10 min of baseline followed by a double-blinded administration of either $100 \%$ oxygen or room air via a nose mask) was repeated.

Oxygen saturation was monitored with a pulse oximeter. Partial oxygen pressure was determined using a blood gas analyser (Radiometer Copenhagen). Conversation was only allowed during the 30-min recovery in order to minimize mental influences. The difference in the R-R intervals in the ECG before and after inhalation of pure oxygen divided by the difference in the oxygen pressures were calculated as the CHRS [ms $/ \mathrm{mmHg}$ ]. A CHRS below $3.0 \mathrm{~ms} / \mathrm{mm} \mathrm{Hg}$ was defined as pathological as reported earlier [9, 10]. Measurements were performed after an overnight fasting period in a noise protected room of constant temperature.

\section{STATISTICAL ANALYSIS}

After testing for normal distribution, group comparisons were calculated using Student t tests or MannWhitney U-tests for continuous variables as appropriate. Categorical data were analysed by $\mathrm{Chi}^{2}$ with Fisher's exact test. Correlations were determined with regression ( $r$ ) analysis coefficient. Multivariate analysis for CHRS prediction was assessed using a multiple regression model. Data are presented as means \pm SEM for continuous variables or as a percentage of patients for categorical variables. A two-sided $\mathrm{P}<0.05$ was considered significant. Statistical analysis was done with SPSS software for windows version 14.0 (SPSS, Chicago, IL). This trial is registered with ClinicalTrials.gov, number NCT00794872.

\section{RESULTS}

Age, gender, cardiovascular risk factors, or concomitant drug therapy did not differed between the control 
and ESRD subjects (Table 1). ESRD resulted from nephrosclerosis $(\mathrm{n}=1)$, polycystic kidney disease $(\mathrm{n}=$ $2)$, suspected glomerulonephritis $(\mathrm{n}=2)$, and hypertensive/vascular renal damage $(n=5)$. The average dialysis vintage was $26 \pm 9$ months. None of the patients had received blood transfusions during the preceding 6 months. Plasma norepinephrine levels were significantly higher in ESRD patients and time-domain HRV parameters indicated a relative predominance of sympathetic activation in ESRD (Table 2).

Table 2. Sympathetic tone, as measured by baseline plasma catecholamine levels and 24-h heart rate variability, in endstage renal disease (ESRD) patients and controls.

\begin{tabular}{lcc}
\hline & $\begin{array}{c}\text { Controls } \\
(\mathrm{n}=10)\end{array}$ & $\begin{array}{c}\text { ESRD } \\
(\mathrm{n}=10)\end{array}$ \\
\hline $\begin{array}{l}\text { Plasma catecholamine levels } \\
\text { Norepinephrine, nmol/L }\end{array}$ & $1.6 \pm 0.4$ & $5.8 \pm 0.6^{*}$ \\
Epinephrine, pmol/L & $342 \pm 75$ & $742 \pm 281$ \\
Heart rate variability & & \\
SDNN, ms & $126.4 \pm 19$ & $100.2 \pm 12^{*}$ \\
SDANN, ms & $121 \pm 20$ & $95.7 \pm 10$ \\
RMSSD, ms & $27.1 \pm 8$ & $15.7 \pm 2^{*}$ \\
Triangular index & $33.6 \pm 4$ & $25.7 \pm 3^{*}$ \\
\hline
\end{tabular}

Values are means \pm SEM. $* \mathrm{P}<0.05$

\section{Chemosensory Deactivation}

None of the patients were hypoxemic at baseline or after oxygen inhalation, with oxygen saturation values ranging from $96 \%$ to $100 \%$. Baseline oxygen saturation and heart rate in patients with ESRD were not significantly different from those observed in controls. After chemoreflex deactivation by inhalation of $100 \%$ oxygen the venous $\mathrm{pO}_{2}$ of all subjects increased from $34 \pm 2$ $\mathrm{mmHg}$ to $65 \pm 2 \mathrm{mmHg}(\mathrm{P}<0.001)$ resulting in an increased R-R interval $(936.9 \pm 19.9$ ms vs. $974.9 \pm 23.3$ ms; $\mathrm{P}<0.001$ ). After room air administration $\mathrm{pO}_{2}$ and heart rate did not change in ESRD patients (Table 3 ) or controls (Table 4) but differed from the values obtained after chemosensory deactivation $(\mathrm{P}<0.05)$ indicating a tonic chemosensory activation in ESRD.

\section{Hyperoxic Chemoreflex Sensitivity}

The hyperoxic CHRS values determined in controls were comparable to those obtained previously in healthy persons [11]. CHRS was significantly impaired in patients with ESRD $(2.9 \pm 0.9 \mathrm{~ms} / \mathrm{mmHg}, \mathrm{P}<0.05)$, as compared with controls $(7.9 \pm 1.4 \mathrm{~ms} / \mathrm{mmHg}$; Figure). A chemoreflex sensitivity below $3 \mathrm{~ms} / \mathrm{mmHg}$ was found in 8 ESRD patients and in 2 controls. No differences were observed in baseline clinical parameters between patients with and without a CHRS below $3 \mathrm{~ms} / \mathrm{mmHg}$. Comparisons of CHRS according to medication (alpha-blockers, beta-blockers, ca-antagonists, ACE-inhibitors/AT-II-antagonists) or history (hypertension, coronary artery disease) failed to reveal any significant difference between those patients.

Table 3. Effects of 100\% oxygen and room air administration in end-stage renal disease patients.

\begin{tabular}{lcccc}
\hline & \multicolumn{2}{c}{$100 \%$ oxygen } & \multicolumn{2}{c}{ Room air } \\
& Before & After & Before & After \\
\hline Heart rate, bpm & $70 \pm 2$ & $67 \pm 2 *$ & $68 \pm 3$ & $67 \pm 2$ \\
R-R interval, ms & $929 \pm 8$ & $970 \pm 29 *$ & $882 \pm 9$ & $895 \pm 10$ \\
PO $_{2}$, mmHg & $33.1 \pm 3.8$ & $50.8 \pm 5.5^{*}$ & $32.7 \pm 2.9$ & $33.1 \pm 3.2$ \\
Oxygen saturation, $\%$ & $97 \pm 1$ & $98 \pm 1$ & $97 \pm 1$ & $97 \pm 1$ \\
MAP, mmHg & $91 \pm 3$ & $89 \pm 2$ & $94 \pm 2$ & $93 \pm 3$ \\
\hline
\end{tabular}

$* \mathrm{P}<0.05$ vs. before oxygen administration. Values are means $\pm \mathrm{SEM}$.

Table 4. Effects of $100 \%$ oxygen and room air administration in controls.

\begin{tabular}{lcccc}
\hline & \multicolumn{2}{c}{$100 \%$ oxygen } & \multicolumn{2}{c}{ Room air } \\
& Before & After & Before & After \\
\hline Heart rate, bpm & $65 \pm 3$ & $62 \pm 2^{*}$ & $65 \pm 2$ & $64 \pm 2$ \\
$\mathrm{R}-\mathrm{R}$ interval, ms & $940 \pm 31$ & $983 \pm 37^{*}$ & $860 \pm 19$ & $860 \pm 19$ \\
$\mathrm{PO}_{2}, \mathrm{mmHg}$ & $36.5 \pm 2.5$ & $43.9 \pm 2.8^{*}$ & $35.7 \pm 2.4$ & $36.2 \pm 2.6$ \\
Oxygen saturation, $\%$ & $98 \pm 1$ & $97 \pm 1$ & $97 \pm 1$ & $97 \pm 1$ \\
MAP, mmHg & $93 \pm 2$ & $92 \pm 3$ & $91 \pm 3$ & $91 \pm 3$ \\
\hline
\end{tabular}

$* \mathrm{P}<0.05$ vs. before oxygen administration. Values are means $\pm \mathrm{SEM}$. 


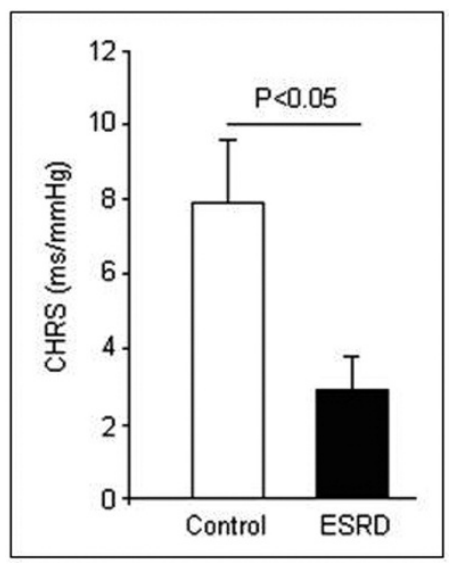

Fig. 1. Hyperoxic chemoreflex sensitivity (CHRS) is impaired in end-stage renal disease (ESRD) patients $(n=10)$ as compared with controls $(n=10)$. Values are means \pm SEM.

\section{Sympathetic Hyperacitivity Predicts Impaired HYPEROXIC CHRS}

The SDANN, as a marker of a relative predominance of sympathetic activity, correlated with the hyperoxic CHRS $(r=0.46 ; \mathrm{P}<0.01)$. On multiple regression analysis $24 \mathrm{~h}$-Triangular index, RMSSD, and plasma norepinephrine levels were independent predictors of an impaired hyperoxic CHRS.

\section{DisCUSSION}

The present study demonstrates that sympathetic hyperactivity influences chemosensory function in ESRD resulting in an impaired hyperoxic CHRS. Our findings support previous reports showing that tonic activation of excitatory chemoreflex afferents contributes to increased systemic efferent sympathetic activity in patients with ESRD [6]. Herein, we have shown for the first time that plasma noradrenaline levels and HRV determine the chemosensory function in these patients. The present study indicates that the hyperoxic CHRS corroborates existing evidence for cardiac sympathetic hyperactivity in ESRD. Several reports demonstrated that cardiac autonomic control is disturbed in ESRD [12-14]. Previous studies demonstrating increased plasma catecholamine levels, abnormal l-metaiodobenzylguanidine (MIBG) kinetics in the heart, and disturbed HRV in ESRD are in line with our findings and may in part explain the impaired CHRS in ESRD, while increased plasma catecholamines can potentiate the chemosensitivity towards oxygen $[15,16]$. CHRS and HRV has been demonstrated to be predictors of ventricular tachyarrhythmias [10]. A combined short-term autonomic reflex testing including CHRS, HRV, and baroreflex sensitivity might improve the prognostic value of these functional modalities but was beyond the scope of the present study. Our results cannot be explained by recruitment of patients from different populations because the ESRD patients and controls were comparable in their demographic characteristics and medical history. This is important since CHRS is altered during heart failure [9, 17], neurocardiogenic syncope [18], and can be influenced by medications likely to modu- late cardiovascular autonomic function [3, 19, 20, 21].

The mechanisms by which ESRD causes autonomic neuropathy are poorly understood. Direct and indirect measures of sympathetic activity agree that an adrenergic overdrive is common in ESRD. It is largely unknown how uremia affects different branches of the cardiovascular autonomic system like sensory afferent and efferent fibres. Chemoreceptor activation appears to contribute to the increased sympathetic drive of patients with ESRD. Currently, we do not know how sympathetic hyperactivity affects chemoreceptor function in ESRD.

There are some limitations of our study. HRV timedomain measures obtained by ambulatory ECG is confounded by daily activities, environment, circadian changes, and medications used. Although we tried to minimize these confounders by detailed exclusion criteria, the interference may still exist. For technical reasons we did not use the more sensitive but at least more invasive MIBG to characterize cardiac sympathetic control. In the current study we assessed chemosenory function in a relatively small number of patients due to the strict exclusion criteria. Finally, autonomic balance is maintained by multiple complex interacting reflex mechanisms, including arterial baroreflex, peripheral chemoreflex, central chemoreflex and pulmonary stretch reflex. Further investigations are needed to explore the exact mechanism of sympathetic chemosensory regulation in ESRD. Herein, we have demonstrated that sympathetic hyperactivity influences chemosensory function in ESRD resulting in an impaired hyperoxic CHRS.

Conflicts of interest: The authors declared no conflicts of interest in relation to this article.

\section{REFERENCES}

[1] Wirta O, Pasternack A, Mustonen J, Laippala P. Renal and cardiovascular predictors of 9-year total and sudden cardiac mortality in non-insulin-dependent diabetic subjects. Nephrol Dial Transplant 1997; 12:2612-7.

[2] Converse RL, Jr., Jacobsen TN, Toto RD, et al. Sympathetic overactivity in patients with chronic renal failure. $\mathrm{N}$ Engl J Med 1992; 327:1912-8.

[3] Ligtenberg G, Blankestijn PJ, Oey PL, et al. Reduction of sympathetic hyperactivity by enalapril in patients with chronic renal failure. N Engl J Med 1999; 340: 1321-8.

[4] Neumann J, Ligtenberg G, Klein II, Koomans HA, Blankestijn PJ. Sympathetic hyperactivity in chronic kidney disease: pathogenesis, clinical relevance, and treatment. Kidney Int 2004; 65:1568-76.

[5] Hausberg M, Kosch M, Harmelink P, et al. Sympathetic nerve activity in end-stage renal disease. Circulation 2002; 106:1974-9

[6] Hering D, Zdrojewski Z, Krol E, et al. Tonic chemoreflex activation contributes to the elevated muscle sympathetic nerve activity in patients with chronic renal failure. $\mathrm{J} \mathrm{Hy-}$ pertens 2007; 25:157-61.

[7] Seals DR, Johnson DG, Fregosi RF. Hyperoxia lowers sympathetic activity at rest but not during exercise in humans. Am J Physiol 1991; 260:R873-R878.

[8] Levey AS, Bosch JP, Lewis JB, Greene T, Rogers N, Roth D. A more accurate method to estimate glomerular filtration rate from serum creatinine: a new prediction equation. Modification of Diet in Renal Disease Study Group. Ann Intern Med 1999; 130:461-70. 
[9] Hennersdorf MG, Hillebrand S, Perings C, Strauer BE. Chemoreflex sensitivity in chronic heart failure patients. Eur J Heart Fail 2001; 3:679-84.

[10] Hennersdorf MG, Niebch V, Perings C, Strauer BE. Chemoreflex sensitivity as a predictor of arrhythmia relapse in ICD recipients. Int J Cardiol 2002; 86:169-75.

[11] Budeus M, Hennersdorf M, Perings C. Chemoreflex sensitivity among patients with paroxysmal atrial fibrillation. Z Kardiol 2003; 92:558-63.

[12] Steinberg AA, Mars RL, Goldman DS, Percy RF. Effect of end-stage renal disease on decreased heart rate variability. Am J Cardiol 1998; 82:1156-8, A10.

[13] Tong YQ, Hou HM. Alteration of heart rate variability parameters in nondiabetic hemodialysis patients. Am J Nephrol 2007; 27:63-9.

[14] Tory K, Suveges Z, Horvath E, et al. Autonomic dysfunction in uremia assessed by heart rate variability. Pediatr Nephrol 2003; 18:1167-71.

[15] Kurata C, Uehara A, Sugi T, et al. Cardiac autonomic neuropathy in patients with chronic renal failure on hemodialysis. Nephron 2000; 84:312-9.

[16] Weil JV, Byrne-Quinn E, Sodal IE, Kline JS, McCullough RE, Filley GF. Augmentation of chemosensitivity during mild exercise in normal man. J Appl Physiol 1972; 33:813-9.

[17] Hennersdorf MG, Perings C, Niebch V, Hillebrand S, Vester EG, Strauer BE. Chemoreflexsensitivity in patients with survived sudden cardiac arrest and prior myocardial infarction. Pacing Clin Electrophysiol 2000; 23:457-62.
[18] Meyer C, Rana OR, Saygili E, et al. Hyperoxic chemoreflex sensitivity is impaired in patients with neurocardiogenic syncope. Int J Cardiol 2009.

[19] Wenzel RR, Allegranza G, Binggeli C, et al. Differential activation of cardiac and peripheral sympathetic nervous system by nifedipine: role of pharmacokinetics. J Am Coll Cardiol 1997; 29:1607-14.

[20] Wallin BG, Sundlof G, Stromgren E, Aberg H. Sympathetic outflow to muscles during treatment of hypertension with metoprolol. Hypertension 1984; 6:557-62.

[21] Neumann J, Ligtenberg G, Oey L, Koomans HA, Blankestijn PJ. Moxonidine normalizes sympathetic hyperactivity in patients with eprosartan-treated chronic renal failure. J Am Soc Nephrol 2004; 15:2902-7.

Corresponding Author:

Christian Meyer, M.D., M.A.

Heinrich-Heine-University Duesseldorf

Department of Medicine

Division of Cardiology, Pulmonology and Vascular Medicine

Moorenstr. 5

40225 Duesseldorf

Germany

Phone: +492118118800

Fax: $\quad+492118118812$

E-mail: Christian.Meyer@med.uni-duesseldorf.de 